\title{
Different Schematic Eyes and their Accuracy to the in vivo Eye: A Quantitative Comparison Study
}

\author{
*Marina Storani de Almeida and $\ddagger$ Luis Alberto Carvalho \\ * Universidade Federal de São Carlos (UFSCar) \\ ¥Grupo de Óptica, IFSC-USP-Instituto de Física de São Carlos, \\ Av. Dr. Carlos Botelho, 1465, \\ 13560-250, São Carlos, SP, Brazil
}

Received on 4 December, 2006

\begin{abstract}
Current ophthalmic technology allows the manipulation of eye components, such as anterior cornea and lens, of the human eye with a considerable precision and customization. This technology opens up the possibility of exploiting some characteristics of the eye in order to improve the methods of correcting optical aberrations. Moreover, product development and research for the eye-care professional has reached very high standards, since there is nowadays software available to design and simulate practically any mechanical or optical characteristic of the product, even before it is thrown into production line. Although quite similar in the general form, different human eye models simulate the image formation by considering different property combinations in the constitutive elements of the eye structure (such as refraction index and surface curvatures), producing retinal images that resemble very closely those of the biological eye. Using optical design software, we have implemented a simulation of 5 well-known schematic eyes available in the literature. These models were the Helmholtz-Laurance, Gullstrand, Emsley, Greivenkamp and Liou \& Brennan. The optical performance of these different models was compared using different quantitative optical quality parameters. The model of Liou and Brennan, contains features of the biological eye that were not considered in previous models, as the distribution of a gradient refraction index and a decentered pupil. Furthermore, it has great reliability since it takes into account the mean value of empirical measurements of the in vivo eye in order to define size and parameters such as anterior and posterior curvature of cornea, lens, axial length, etc. Comparisons between the MTF (Modulation Transfer Function), spot diagrams and ray fan showed the difference in image quality between eye models, and the Strehl Ratio was also used as a parameter of comparison. A careful comparison between the different models showed that the first four schematic eyes have better optical quality than what is expected for the general and healthy emmetropic in vivo eye. Liou and Brennan schematic eye is the one that most closely resembles the in vivo biological eye. Therefore, in applications, such as research or product development for customized vision correction, which must consider optical properties intrinsic to the biological eye, we recommend this latter model; for applications that do not require refraction-limited performance, most of the other models should be a good approximation.
\end{abstract}

Keywords: Eye model; Schematic Eye; Ray tracing; Optical aberrations

\section{INTRODUCTION}

The interest in the physiological aspects and optical properties of the human in vivo eye, and how they relate to visual acuity, come from very ancient times. Historically, after Gauss (1841) established the basic laws that govern image formation properties, many theoretical models have been proposed. In the late $19^{\text {th }}$ century, Helmholtz undertook a very thorough study on this subject, and published the now famous collection Helmholtz Treatise on Physiological Optics[1]. This model was later modified by Laurance and became known as the Helmholtz-Laurance model[2], which contains all optical surfaces found in the biological eye. Although this model designates refractive indices to eye components that not necessarily correspond to true measured values, its overall properties have a close resemblance to those of the human eye. Afterwards, the Swedish Ophthalmologist Allvar Gullstrand (1862-1930) conducted important research in the field of physiology and in 1911 received the Nobel Prize for his work regarding the eye as an optical element [3]. While Gullstrand's simplified schematic eye treats the cornea as a single refracting surface, just as the previous model from Helmholtz-Laurence, in Gullstrand's non-simplified model the cornea is considered to have two surfaces, which guar- antees a perfect image formation at the retina. Although it simplifies the cornea, the vitreous and the aqueous humor, this model is especially suitable for the computation of intraocular lens (IOL) power, as it also contains the anterior and posterior surface of the crystalline lens.

One of the simplest eye models available is the Emsley schematic eye [4], since it contains just a single refractive surface. Due to its simplicity, it is widely used in undergraduate courses in optometry, ophthalmology and vision science. The improvement of corneal topography techniques in the late 80 s and along the 90s [5-14] allowed the inclusion of more precise corneal surface data into schematic eye models. This represented an important progress in ophthalmology, as the cornea contributes to approximately $2 / 3$ of the overall refractive power of the eye. Relying on these techniques, in 1995 Greivenkamp and colleagues proposed an eye model [15] containing four refracting non-spherical surfaces that considered retinal contrast sensitivity, refraction-limited properties, among others. From this time up to modern days an incredible amount of techniques and instrumentation for visual quality measurements were implemented. Among them are the highly successful application of the Hartmann-Shack wavefront sensor [16-19] and other sensor with different symmetries [20], to measure higher order aberrations of the human 
eye, which are described by a set of Zernike Polynomials [2122]. There is also the double-pass technique [23] which can be used to measure even higher frequency aberrations associated with scatter. Together these techniques and instrumentation form a collection of tools that aid the eye-care professional in providing the best diagnostic and treatment available to their patients. Although these eye models are of great use to the development of such techniques, current eye models are limited since they do not consider all the complexity of the visual system, once the properties of each component are approximations based on the biological eye. Hence, the ongoing interest in coming up with better eye models, which is essential to guide the refraction correction methods already in use, improve them and even allow the development of new ones. In 1997 Liou \& Brennan have proposed an interesting model [24], which is, to our knowledge, the closest to anatomical, biometric and optical data as compared to the physiological eye. Their objective was to develop a model that could be used to predict visual performance under normal and altered conditions of the eye, using empirical values of ocular parameters.

All of the models above have their own merit on describing the optical and physiological properties of the human eye. The present work should serve as a guide for precise quantitative comparison between some of the schematic eyes available in literature. Depending upon the application/research that will be implemented, one or other eye should have more desirable features than the other, varying from an extremely simple and approximate eye model to more sophisticated and anatomically correct ones

\section{MATERIAL AND METHODS}

The quantitative comparison between the five eye models considered here was based on simulations implemented using the Zemax software package [25]. This software was chosen taking into account the authors' previous experience and the availability of this tool in our research laboratory. However, there are other software such as CodeV (www.opticalres.com) and OSLO (www.lambdares.com) where the current analysis can also be undertaken. In Fig. 1 we list the layouts of the models analyzed.

Observing the layouts we notice that the HelmholtzLaurance and the Gullstrand models are very similar, having little differences in corneal radius of curvature, size and index of refraction. Actually, the Gullstrand model is a slight improvement of the Helmholtz-Laurance eye model. The Emsley eye is the simplest model, as it considers the entire eye as composed of just one material. On the other hand, the Gullstrand and Liou \& Brennan models are the most complex ones, as they take into account some aspects of the physiological eye, such as the shape of the posterior cornea, to evaluate the ray tracing. Besides considering the gradient variation of the refraction index, Liou \& Brennan model considers decentration and tilt of the pupil that are present in the biological eye for an entrance pupil diameter of $4 \mathrm{~mm}$. Recent research into pupil geometric center has revealed that this feature changes with change in illumination and pupil size [26-28], although this was not taken into account in our simulations. The first four models presented on Table 1 were proposed for paraxial rays with no restriction on the entrance pupil diameter (EPD) value. However, the Liou \&Brennan model [24] was proposed for an EPD of $4 \mathrm{~mm}$ and a distance between the eye and the object of approximately $44 \mathrm{~cm}$. Other aperture values would certainly modify the shape and the refraction index of the crystalline lens. Even though, we conducted the simulation using the four aperture values considered for the other models using the same properties for the crystalline lens. As mentioned before, certain optical engineering functions were used for performance analysis and comparison between the presented models. Among them the Modulation Transfer Function (MTF), the Spot Diagram, the Ray Fan and the Strehl Ratio, which are described in detail in the following section.

\section{RESULTS}

\section{Modulation Transfer Function}

The sharpness and contrast of an imaging system or of a component of the system may be characterized by a parameter called the Modulation Transfer Function (MTF), also known as spatial frequency response. The MTF curve has different meanings according to the corresponding frequency.Its height at frequencies of 1.5 cycles/degree represents the contrastbehavior of the optical system [29]. It is known from experience that a good lens system should perform over $95 \%$ at this frequency for both sagittal and tangential directions, and values worse than $90 \%$ represents a bad performance.

Frequencies in the gap of 3 to 12 or higher cycles/degree represent the sharpness-ability of a lens. MTF readings taken at 12 cycles/degree indicate how good a lens can transmit very fine structures. For an optimal quality based on the human eye, the lens should perform over 50\% at 6 cycles/degree [29]. Perceived image sharpness is more closely related to the spatial frequency where MTF is $50 \%(0.5)$, where contrast has dropped by half. Typical $50 \%$ MTF frequencies are in the vicinity of 12 to 24 cycles/degree for individual components and often as low as 9 cycles/degree for entire imaging systems [29].

All the tangential and sagital MTF curves obtained for all 5 models are presented in Fig. 2, and they are organized in increasing values of EPD. The tangential and the sagittal modulation transfer functions were plotted to illustrate the influence of decentration and tilt of the pupil considered by the Liou \& Brennan model.

\section{Spot diagram}

The spot diagram is a two dimensional distribution of ray intersections at the image plane. It is the result of off-axis rays that are launched to hit every point on a square grid placed over the entrance pupil of the system and that, after refraction, hit the image plane[25]. When the lens aper- 


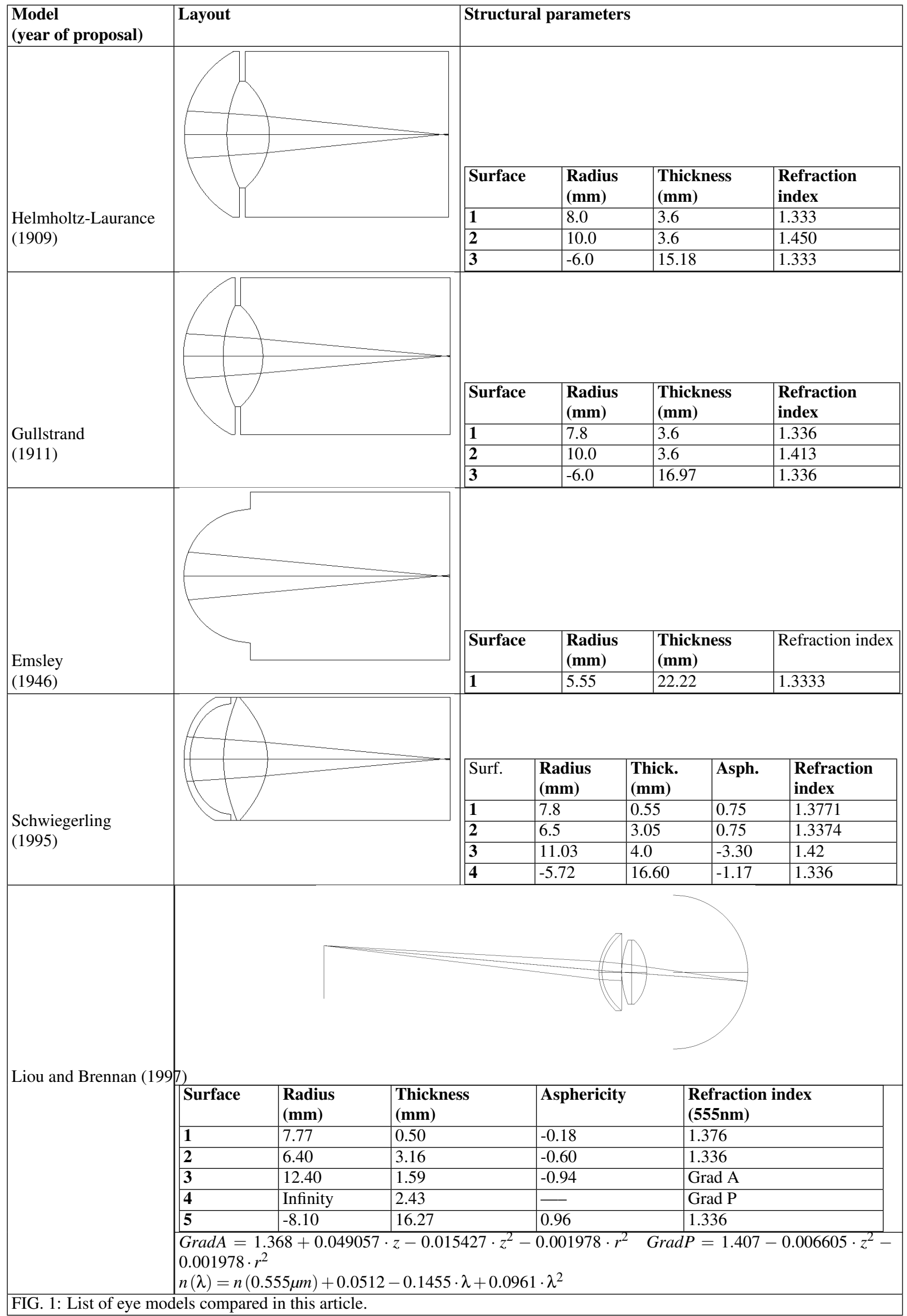




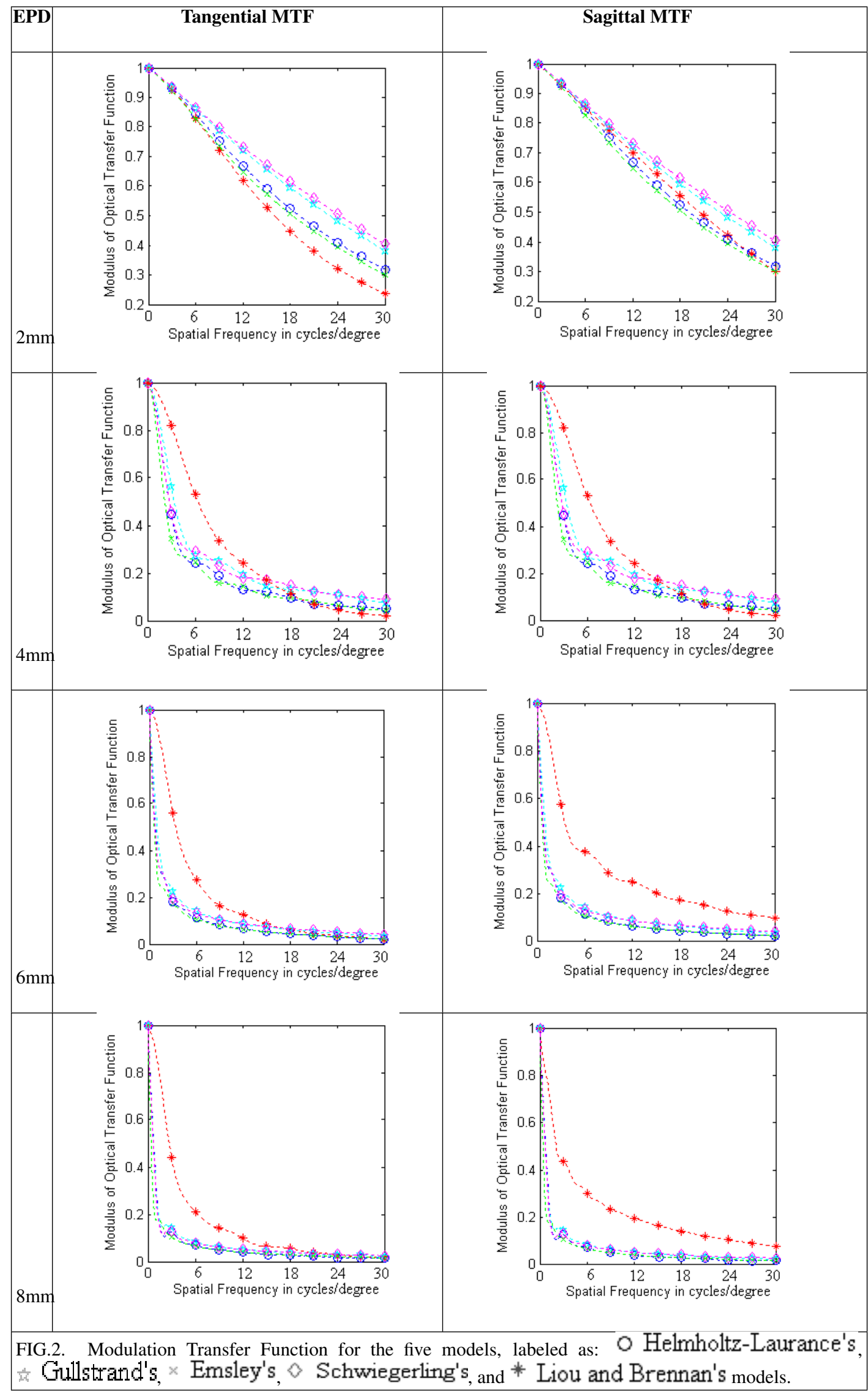




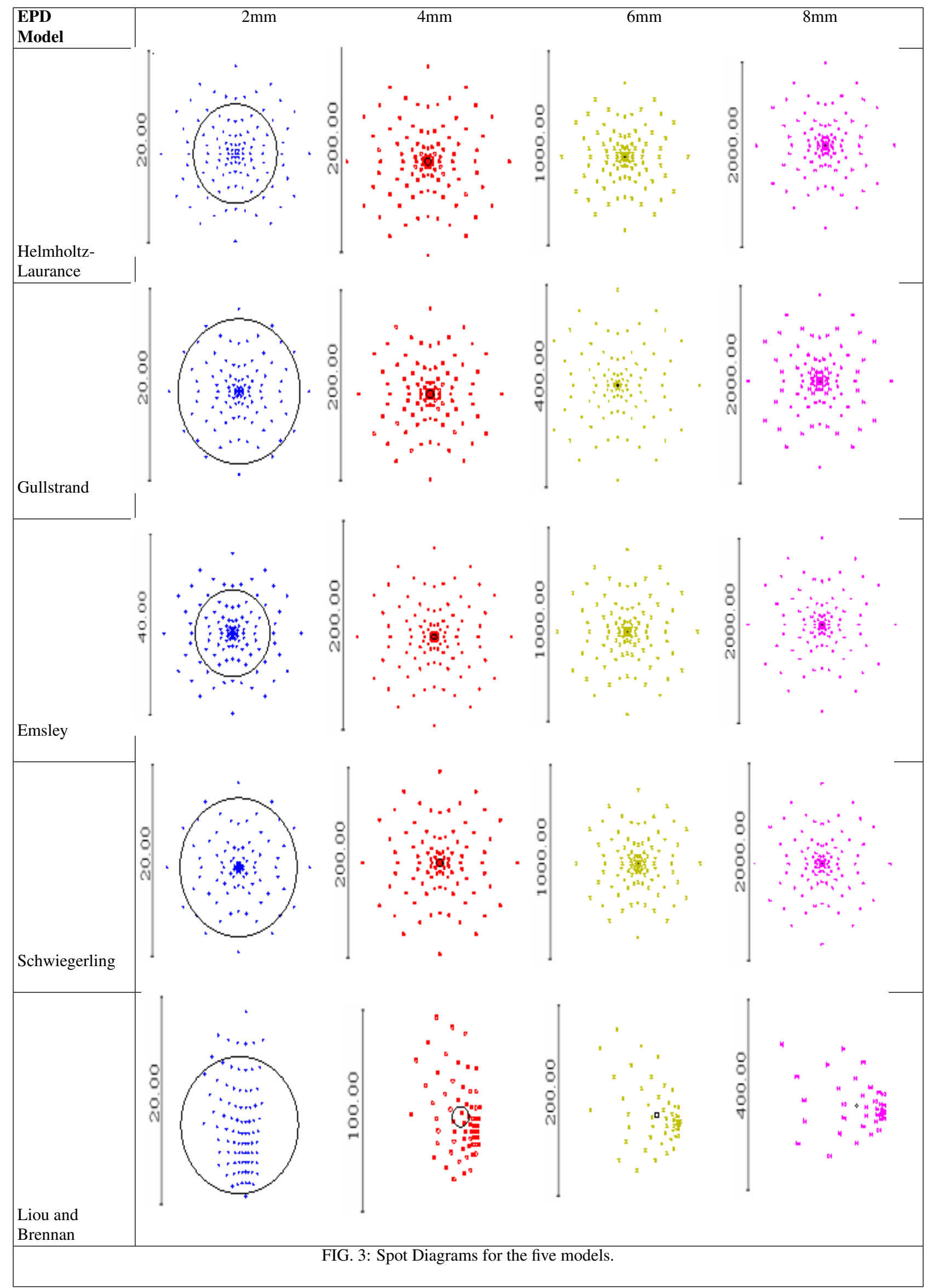


TABLE 1. Values for RMS radius, geometric radius and Airy disc diameter, in microns, for each eye model for an EPD of $4 \mathrm{~mm}$.

\begin{tabular}{|l|c|c|c|}
\hline \multicolumn{1}{|c|}{ Model } & RMS Radius & Geometric Radius & Airy Diameter \\
\hline Helmholtz & 31.843 & 63.182 & 4.863 \\
\hline Emsley & 40.376 & 81.156 & 5.307 \\
\hline Gullstrand & 26.373 & 58.165 & 5.458 \\
\hline Schwiegerling & 32.849 & 67.546 & 5.403 \\
\hline Liou and Brennan & 11.007 & 28.660 & 5.131 \\
\hline
\end{tabular}

ture is small, diffraction effects may be more important than lens aberrations in limiting the sharpness of images. When light passes through a small circular aperture, a diffraction pattern is formed consisting of a bright central circle, also known as the airy disk, and low contrast peripheral concentric rings [30]. When the eye focuses on a point object, the image formed at the retina will be a circle with the size of the airy disk, even in the ideal case of aberration absence. The airy disk diameter is given by equation 1 .

$$
D=\frac{2.44 \lambda}{d}
$$

Where $\lambda$ is the wavelength and $d$ is is the diameter of the system aperture. Since the airy disk diameter is proportional to the diameter of system aperture, the effects of diffraction increase as the EPD decreases. Wave-front aberrations are the limiting factor in image quality at large apertures and diffraction sets the limit at small apertures. The construction of spot diagrams is one of the methods for visualizing the effect of aberrations have on image quality. The distribution of ray intersections depends on the aberrations of the system. For our analysis, a square grid pattern was selected at the EPD for ray tracing, where the principle ray is always at the center of the pattern. The airy disk is shown as a black circle for each spot diagram (see Fig. 3).

The root mean square (RMS) spot radius is another measure of quality that relies on the spot diagram. It is computed as the RMS of all distances between each marginal ray intersection $\left(x_{i}, y_{i}\right)$ with the image plane and a reference point $\left(x_{o}, y_{o}\right)$, generated by the chief ray intersection. The RMS spot radius is given by

$$
R_{r m s}=\frac{\sum_{i=1}^{n} \sqrt{\left(x_{i}-x_{0}\right)^{2}+\left(y_{i}-y_{0}\right)^{2}}}{n}
$$

Where $n$ is the total number of rays considered.

Another value obtained from the spot diagram is the geometric radius, which just encloses the outermost ray pierce in the pattern relative to the reference point. The values obtained for RMS radius, geometric radius and the airy disk diameter obtained for each model for an EPD of $4 \mathrm{~mm}$ are presented in micrometers on Table 1 and Fig. 4.

\section{Ray Fan}

Another technique of analysis is the ray fan plot, which describes the relative distances of the rays that hit the image plane. This diagnostic plot is obtained from an off-axis ray launched along the EPD. The chief ray is the one that pierces exactly at the center of the EPD, and its height on the image plane is the reference for the others rays. The height that rays hit the EPD is normalized and placed at the horizontal axis, while the relative distance of the rays that arrive at the image plane is placed on the vertical axis.

The shape of the ray fan plot depends upon the type and magnitude of the aberrations present in the system. An ideal system would convert all the rays to a unique point on the image plane, and its corresponding ray fan would be a line exactly over the abscissa.

\section{Strehl ratio}

The Strehl ratio is defined as the ratio of the central intensity in the aberrated pattern to the central intensity in the un-aberrated pattern. Unless a design for an imaging system approaches diffraction-limited performance, there is no point in looking at Strehl values [25]. The Strehl ratio ranges from 0 (for systems having significant aberration) to 1 (for perfect systems). In Table 5 and Fig. 5 it is shown the Strehl ratio values for each eye model.

\section{DISCUSSION}

The five eye models presented here were simulated using the Zemax software package, based on parameters for each eye model. The retinal images were analyzed by conventional optical design test functions. Comparisons between the MTF, spot diagram and ray fan showed the difference in image quality for the considered models. The Strehl ratio was also used for comparison.

From Fig. 2 we notice that although all the schematic eyes have a good contrast, i.e., have reasonable values of MTF for a spatial frequency of 1.5 cycles/degree, the image sharpness is not good for all models. Considering that the physiological eye has a MTF of approximately 0.3 for a spatial frequency of 


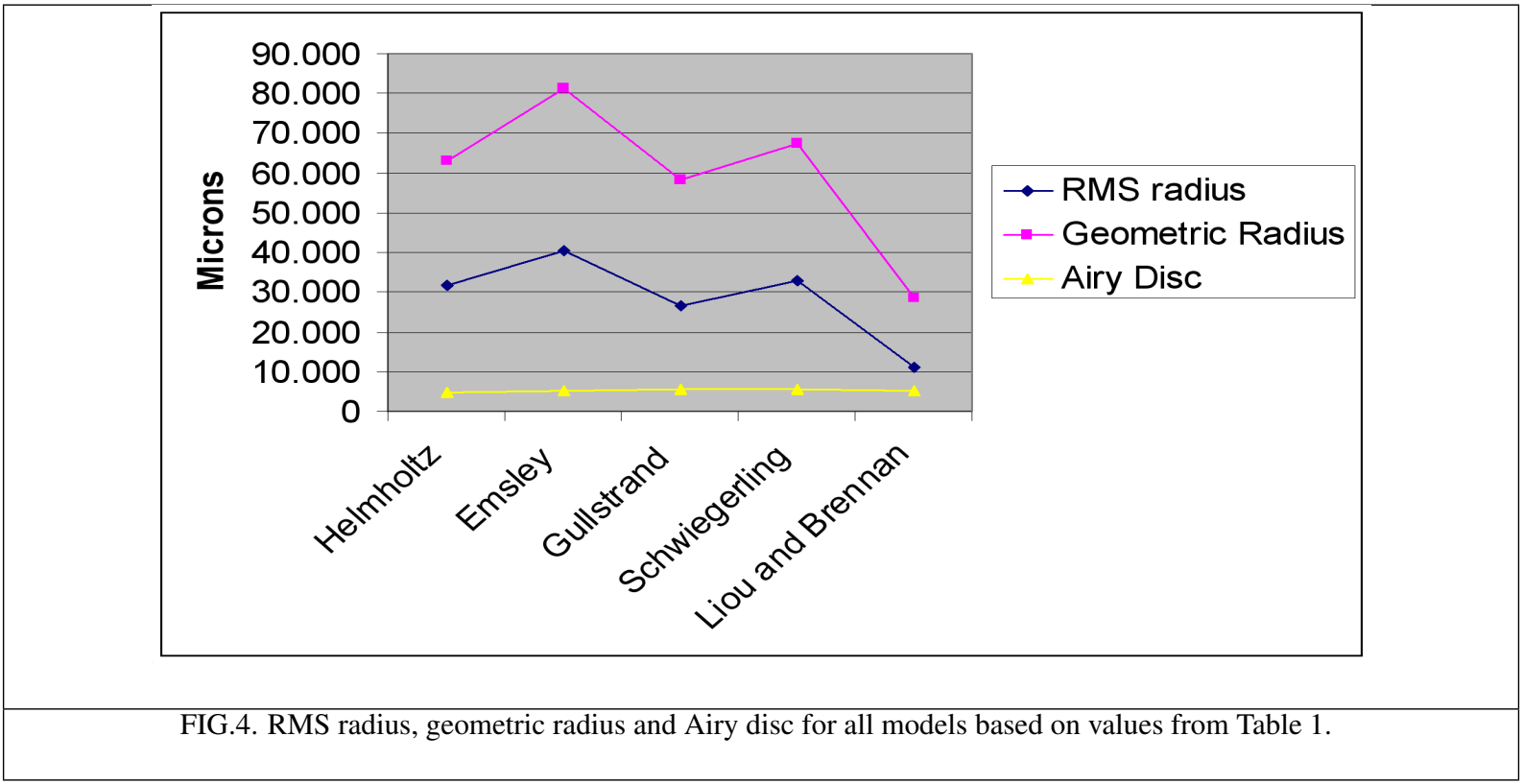

TABLE 5: The Strehl ratio for each model and EPD considered in the analysis.

\begin{tabular}{|l|l|l|l|l|}
\hline $\begin{array}{l}\text { EPD } \\
\text { Model }\end{array}$ & $2 \mathrm{~mm}$ & $4 \mathrm{~mm}$ & $6 \mathrm{~mm}$ & $8 \mathrm{~mm}$ \\
\hline Helmholtz & 0.795 & 0.028 & 0.011 & 0.039 \\
\hline Emsley & 0.826 & 0.033 & 0.015 & 0.013 \\
\hline Gullstrand & 0.938 & 0.066 & 0.017 & 0.043 \\
\hline Schwiegerling & 0.961 & 0.065 & 0.019 & 0.014 \\
\hline Liou and Brennan & 0.820 & 0.158 & 0.045 & 0.038 \\
\hline
\end{tabular}

15 cycles/degrees [31], Liou \& Brennan schematic eye is the one that most closely resembles the in vivo human eye. It is interesting to note that the tangential and sagittal MTFs of the Liou \&Brennan model are the only ones that are different for all the considered apertures, something that does not happen for the other models. The reason for this difference between the tangential and the sagittal MTF on the Liou and Brennan model is that, although the object is on-axis, it is not rotationally symmetric about the axis of the eye (it considers decentration and tilt of the pupil that are present in the biological eye), which means that the MTF will not be rotationally symmetrical. Another point to be considered is that in general the MTF curves get worse as the aperture is increased, since the models furnish increasing spherical aberration as the aperture increases. A good measure that would consider all meridians at once is the volume MTF [32], which was not implemented at this time.

From Fig. 3 we observe that all the spot diagrams differ from the ideal case, which in the ideal case would be just a point in the image plane. The Airy disk indicates the occurrence of the diffraction effect on the images. Spot diagrams that are more compact indicate less aberration. ¿From the spot diagrams, it is easy to see that the Liou \& Brennan model has the best performance for an entrance pupil diameter of $4 \mathrm{~mm}$.

Analyzing the ray fan plots in Fig. 4, we observe an evolution from the Helmholtz to the Liou and Brennan schematic eye, i.e., as abscissa values increase the image size values do not increase as much as in the other models, demonstrating that the Liou \& Brennan model contains less spherical aberration . All of the models are good at the central portion of the image plane, at the intersection of the principal ray, but, as expected, marginal rays are the most aberrated. The Gullstrand and Emsley ray fans are the most similar ones, but observing them very carefully we see that the plot for the Gullstrand model has a greater length on the abscissa axis. The Schwiegerling model plot looks like if the system has a spherical aberration that is compensated by other aberrations.

Finally, the Strehl ratio was computed for all models (Table 5 and Fig. 5). It may be seen that the Strehl ratio decreases as the EPD increases. This decreasing tendency is slower for the Liou and Brennan model, which means that this model is closer to the diffraction limit for a greater range of EPDs. It is not recommended to calculate the Strehl ratio when the MTF plot is distant from the diffraction limit, as its values will be so small that can simply be ignored. However, its values are shown on Fig. 5 in order to illustrate the rate of growing aberrations for the models considered, and are also a factor of comparison. Although the Liou \& Brennan model does not have the greatest Strehl ratio for an EPD of $2 \mathrm{~mm}$, this is the model that has the greatest values of Strehl ratio for the apertures of 4 and $6 \mathrm{~mm}$ (it is important to remember here that this model was proposed for an EPD of $4 \mathrm{~mm}$ ).

A careful comparison of the modulation transfer function of the five models shows that the first four schematic eyes provide images with higher quality than what is expected for the general human eye. The Liou and Brennan's model does not 


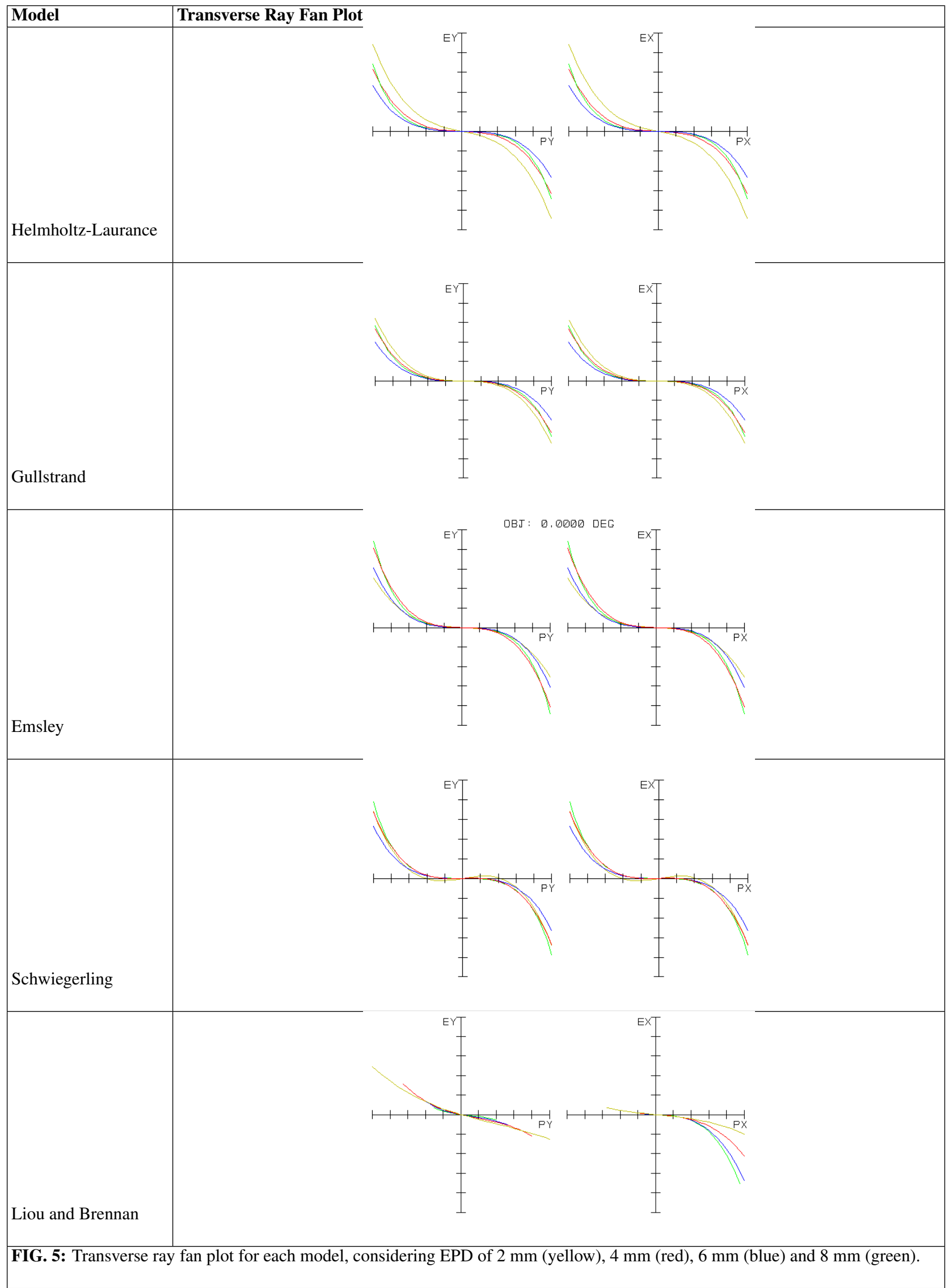




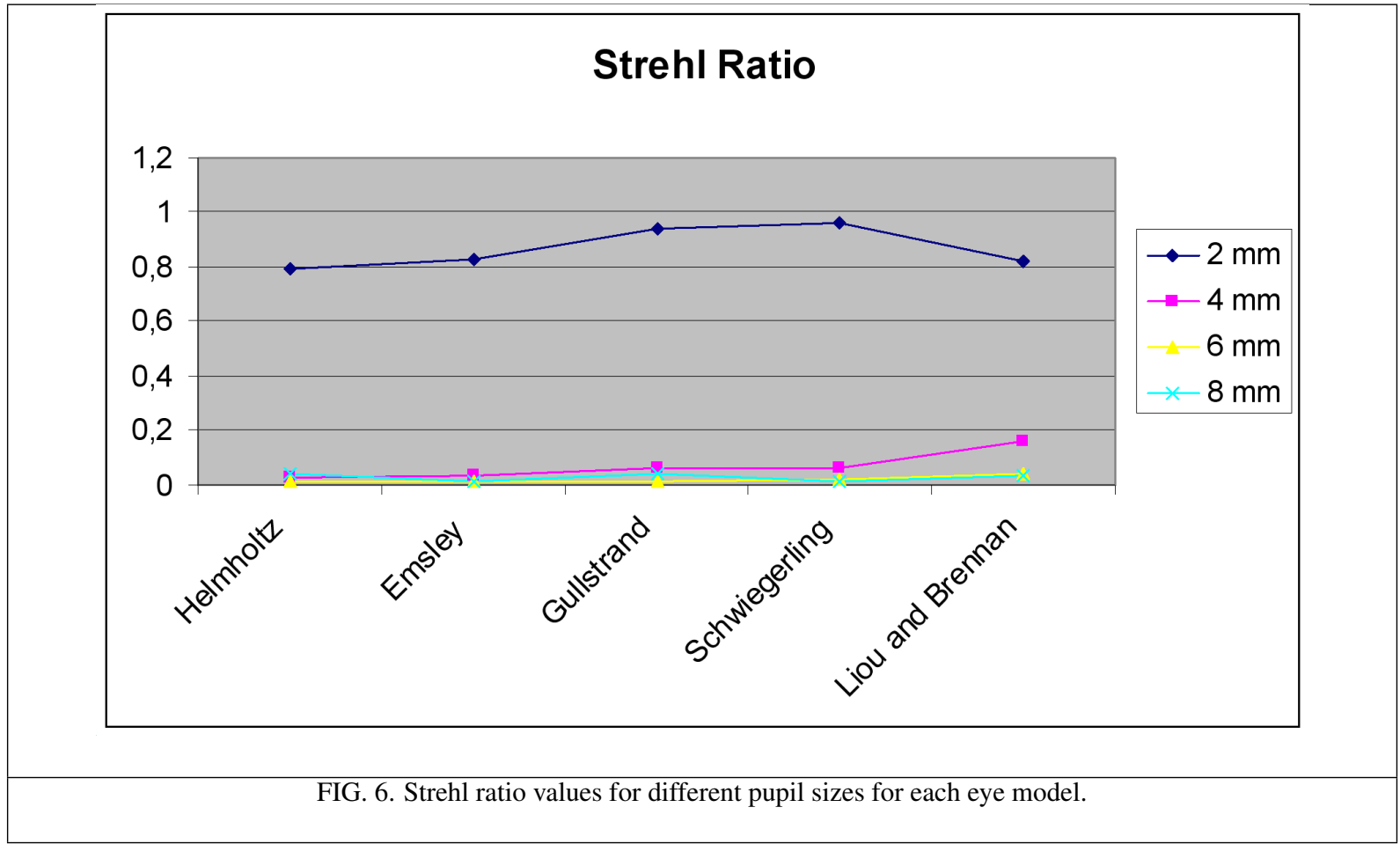

have the best MTF, but is the one that most closely approximates to the in vivo human eye. The other optical design test functions (Spot Diagram, Ray Fan and Strehl ratio) showed the best results for the Liou and Brennan schematic eye. Another type of comparison, which was not carried out here, but that would certainly add value to the other types of optical quality parameters used, is the Zernike Polynomial fitting of the wavefront for each eye model. A comparison of each Zernike coefficient would clearly and quantitatively indicate the benefits of each model in terms of individual aberrations.

In general terms we may claim that the Liou and Brennan's schematic eye would be the best choice for applications which require eye models that are optically and physically more similar to the biological eye. Otherwise, most of the other simplified model eyes should be sufficient for applications which require only an approximation of the optical and physical properties of the eye.
[1] H. von Helmholtz Helmholtz's treatise on physiological optics Edited by J. P. C. Southall (2000).

[2] M. Alpern, The eyes and vision. In: Handbook of Optics (1978).

[3] A. H. Tunnacliffe, Introduction to Visual Optics. (1989).

[4] L. S. Pedrotti and F. L. Pedrotti, Optics and Vision (1998).

[5] D. J. Schanzlin and J. R. Robin, Corneal topography: measuring and modifying the cornea. (1992).

[6] D. R. Sanders and D. D. Koch, An atlas of corneal topography. (1993).

[7] S. D. Klyce, Invest. Ophthalmol. Vis. Sci. 25, 1426 (1984).

[8] D. D. Koch, G. N. Foulks, C. T. Moran, and J. S. Wakil, Refract. Corneal Surg. 5, 424 (1989).

[9] J. Wang, D. A. Rice, and S. D. Klyce, Refract. Corneal Surg. 5, 379 (1989).

[10] R. J. Mammone, M. Gersten, D. J. Gormley, R. S. Koplin, and V. L. Lubkin, IEEE Trans. Biomed. Eng. 37, 66 (1990).

[11] P. P. van Saarloos and I. J. Constable. Optom. Vis. Sci. 68, 960 (1991).

[12] R. B. Mandell, Contact Lens Assoc. Ophthalmol. J. 18, 267 (1992).

[13] L. Carvalho, S. A. Tonissi, and J. C. Castro, J. Cataract Refrac. Surg. 25, 821 (1999).
[14] L. A. Carvalho, M. Stefani, A. C. Romão, L. Carvalho, J. C. Castro, S. Tonissi, P. Schor, and W. Chamon, J. Cataract Refrac. Surg. 28, 2006 (2002).

[15] J. E. Greivenkamp, J. Schwiegerling, J. M. Miller, and M. D. Mellinger, Am. J. Ophthalmol. 120, 227 (1995).

[16] J. Liang, B. Grimm, S. Goelz, and J. F. Bille, J. Opt. Soc. Am. A Opt. Image Sci. Vis. 11, 1949 (1994).

[17] L. A. Carvalho, A. C. Romão, M. Stefani, L. A. Carvalho, J. C. Castro, F. Yasuoka, F. Scannavino Júnior, J. Santos, P. Schor, and W. Chamon. Arq. Bras. Oftalmol. 66, 261 (2003).

[18] L. A. V. Carvalho and J. C. Castro, Braz. J. Phys. 33, 140 (2003).

[19] L. A. Carvalho, J. Biomed. Inform. 37, 1 (2004).

[20] L. A. Carvalho, J. Castro, W. Chamon, and P. Schor. J. Refract. Surg. 22, 954 (2006).

[21] M. Born and E. Wolf, Principles of Optics: Electromagnetic Theory of Propagation, Interference and Diffraction of Light. 7th ed. (1999).

[22] L. A. Carvalho, Proceedings of SPIE 5772, 181 (2005).

[23] F. Diaz-Douton, A. Benito, J. Pujol, M. Arjona, J. L. Guell, and P. Artal, Invest. Ophthalmol. Vis. Sci. 47, 1710 (2006).

[24] H. L. Liou and N. A. Brennan, J. Opt. Soc. Am. A 14, 1684 
(1997).

[25] J. M. Geary, Introduction to lens design with practical ZEMAX®examples. (2002).

[26] G. Walsh, Ophthalmic. Physiol. Opt. 8, 178 (1988).

[27] M. A. Wilson, M. C. W. Campbell, and P. Simonet, Optom. Vis. Sci. 69, 129 (1992).

[28] L. A. Carvalho and A. C. Romão, Revista de Física e Instrumentação 16, 24 (2003).
[29] N. Koren, Understanding image sharpness part 1: Introduction to resolution and MTF curves. Available on: ¡http://www.normankoren.com, , accessed in 12 mar. 2006.

[30] M. M. Sternheim and J. W. Kane, General Physics. 2nd ed. (1991).

[31] F. W. Campbell and R. W. Gubisch, J. Physiol. 186, 558 (1966).

[32] K. Y. Li and G. Yoon, Optics Express. 14, 12552 (2006). 